\title{
DIFICULDADES NA AMAMENTAÇÃO NO PRIMEIRO MÊS DE VIDA: IMPACTO DOS CONTEXTOS DE VIDA
}

\author{
Paula Nelas \\ Emília Coutinho \\ Cláudia Chaves \\ Odete Amaral \\ Carla Cruz \\ Escola Superior de Saúde de Viseu - CI\&DETS \\ pnelas@gmail.com \\ https://doi.org/10.17060/ijodaep.2017.n1.v3.987
}

Fecha de Recepción: 25 Marzo 2017

Fecha de Admisión: 1 Abril 2017

\section{RESUMO}

Enquadramento: 0 aleitamento materno não é determinado simplesmente de forma biológica, também envolve fatores emocionais e socioculturais.

Objetivos: Identificar as dificuldades mais frequentes associadas à amamentação no primeiro mês de vida do bebé; avaliar a relação entre as dificuldades na amamentação e a satisfação com a vida; analisar a relação entre as dificuldades na amamentação e as variáveis sociodemográficas.

Métodos: Estudo quantitativo, descritivo, correlacional. Os dados foram recolhidos através de um questionário, aplicado em dois momentos, aos 7 e aos 30 dias de vida do bebé, que permitiu fazer a caracterização sociodemográfica, caracterização da amamentação. Inclui ainda a Escala de Satisfação com a Vida (Simões, 1992). A amostra é não probabilística por conveniência, constituída por 255 puérperas que amamentam, com idade média de 20,7 anos, inscritas nas Unidades de Saúde Familiar e Unidades de Cuidados de Saúde Personalizados na região centro de Portugal.

Resultados: As participantes são maioritariamente casadas, com escolaridade inferior ao $3^{\circ}$ ciclo, empregadas em tempo completo e residentes na cidade. Quanto às dificuldades mais frequentes associadas à amamentação nos dois momentos de avaliação do estudo ( $7^{0}$ e e $30^{\circ}$ dia de vida do bebé) verificamos que as dificuldades/problemas sentidas ao $7^{\circ}$ dia se mantêm no 30ำ, predominando as fissuras $(77.5 \%)$, o ingurgitamento mamário $(66.7 \%)$, a mastite $(63.6 \%)$, as dificuldades na pega (82.4\%), na posição e postura para amamentar (50.0\%). As dificuldades na amamentação não se relacionam com a satisfação com a vida.

Conclusões: Sabendo que as mulheres apresentam dificuldades relacionadas com a amamen- 
tação é importante 0 apoio por parte dos enfermeiros de modo a que as mesmas possam ultrapassá-las e que não se constituam como motivo de abandono da amamentação.

Palavras-Chave: Amamentação; Dificuldades; Manutenção; Satisfação com a vida.

\section{ABSTRACT}

Difficulties in breastfeeding in the first month of life: life contexts impact

Autores: Paula Nelas, Emília Coutinho, Cláudia Chaves, Odete Amaral, Carla Cruz

Background: Breastfeeding is determined not just organically but also involves emotional and socio-cultural factors.

Objectives: Identify the most frequent difficulties associated with breastfeeding in the first month of life of the baby; Evaluate the relationship between difficulties in breastfeeding and satisfaction with life; Analyze the relationship between difficulties in breastfeeding and sociodemographic variables.

Methods: A quantitative study, descriptive, correlational. The data were collected through a questionnaire, applied in two moments, at 7 and 30 days of the baby's life, which allowed the sociodemographic and breastfeeding characterization. It also includes Satisfaction with Life Scale (Simões, 1992). The sample is not probabilistic for convenience, made up of 255 mothers who breastfeed, entered in the Family Health Units and Custom Health Care Units in the central region of Portugal.

Results: Participants have an average age of 20.7 years, mostly married, with education less than the 3rd cycle, and residents employed full time in the city. As for the most common problems associated with breastfeeding in the two stages of evaluation that women had the 7th day and continued to be the 30th day, the fissures predominate $(77.5 \%)$, breast engorgement $(66.7 \%)$, mastitis $(63.6 \%)$ the difficulties in the handle $(82.4 \%)$, the position and posture for nursing $(50.0 \%)$. The difficulties in breastfeeding are not related to satisfaction with life.

Conclusions: In view of these results, and knowing that women have difficulties with breastfeeding in the two time points, it is important the support from nurses so that they can overcome them and do not constitute a repudiation of reason breastfeeding.

Keywords: Breastfeeding; Difficulties; Maintenance; Life Satisfaction.

\section{INTRODUÇÃO}

A Organização Mundial da Saúde recomenda o aleitamento materno exclusivo até ao sexto mês de vida e complementado até aos dois anos ou mais, sustentando-se nos benefícios que o mesmo tem, essencialmente, em termos de saúde e bem-estar do bebé e da mãe (Pinto, 2008). Em Portugal, o Plano Nacional de Saúde também aconselha o estímulo desta prática, assumindo-a como um critério de qualidade dos cuidados de saúde perinatais.

Apesar de na atualidade 0 aleitamento materno ser encarado como a melhor alternativa para 0 recém-nascido e muito se ter investido para que este constitua o único alimento até aos seis meses de idade, em Portugal, apesar das recomendações, continuam-se a verificar elevadas taxas de abandono precoce, pois apenas $55-64 \%$ das mães amamentam aos três meses e $34 \%$ aos seis meses (Aguiar \& Silva, 2011).

A amamentação deve ser ativamente estimulada pelos profissionais de saúde envolvidos na relação mãe, filho e família. 0 aconselhamento deve ser iniciado durante a gravidez, uma vez que muitas mulheres tomam a sua decisão nessa altura, devendo ser mantido após o nascimento, facultando à mãe um apoio contínuo para que se possam esclarecer dúvidas, desmistificar falsas crenças, medos, tabus e receios (Greiner, 2014). 
Mesmo estando informadas da importância do aleitamento materno, muitas mulheres não amamentam porque se deparam com dificuldades, sobretudo nos primeiros dias pós-parto, ou não têm sucesso na sua manutenção devido a problemas específicos do aleitamento materno, como sejam traumas mamários, mamilos invertidos, pega incorreta, dor, desconforto, entre outros (Cremonese, L., Wilhelm, L.A., Santos, C. dos; Alves, C.N., Martello, N.V., Silva, S.C. da, Castiglioni, C.M., \& Ressel, L.B. (2011). Estas dificuldades são geralmente pequenas e podem ser facilmente superadas com aconselhamento, assistência e suporte (Walker, 2008). No entanto, problemas que parecem menores para os profissionais de saúde podem ser questões importantes para a mulher. 0 apoio adequado na comunidade e na prática, essencialmente, através de profissionais de saúde treinados, é fundamental para minimizar o impacto dos problemas da amamentação (Walker, 2008).

Também têm sido enumerados um conjunto de fatores que se direcionam para a decisão de amamentar, tais como as condições psíquicas da mãe, que abarcam as experiências anteriores e 0 significado atribuído à amamentação, as condições biológicas da mãe, sobretudo o seu estado de saúde, a presença de dor ou doenças, as representações sociais sobre a amamentação, que dizem respeito ao valor cultural que a sociedade confere a este processo, a satisfação da própria mulher com a vida, o apoio social, que corresponde aos mecanismos com que a mulher pode contar para a manutenção da amamentação, especificamente 0 apoio por parte dos serviços de saúde e da família (Greiner, 2014).

Também assume importância a motivação e a satisfação da mulher perante a vida, na medida em que são estes dois fatores que dinamizam, direcionam e condicionam o comportamento. Quanto mais motivada estiver a mulher em relação à amamentação e quanto mais satisfação a mesma revelar em relação à própria vida, maior será o sucesso da amamentação.

A satisfação com a vida é um julgamento cognitivo de alguns domínios específicos na vida da pessoa, tais como, a saúde, o trabalho, as relações sociais, a autonomia entre outros, ou seja, tratase de um processo de juízo e de avaliação geral da própria vida, segundo um critério próprio. A satisfação com a vida reflete o bem-estar subjetivo individual, o modo e os motivos que levam as pessoas a viverem as suas experiências de vida de uma forma positiva. Desta feita, a opção pela amamentação e a superação das dificuldades a ela inerentes poderão depender da satisfação com a vida.

\section{METODOLOGIA}

0 estudo realizado é quantitativo, longitudinal, descritivo e correlacional, com uma amostra não probabilística por conveniência, sendo critério de inclusão puérperas que amamentam, nos dois momentos de colheita de dados ( $7^{\circ}$ e $30^{\circ}$ dia após o nascimento), inscritas nas Unidades de Saúde Familiar (USF) e Unidades de Cuidados de Saúde Personalizados (UCSP) em duas cidades do centro de Portugal, que recorreram às consultas de vigilância de saúde infantil e consultas de enfermagem. A amostra perfaz 255 participantes com uma idade mínima de 17 anos e uma máxima de 42 anos, correspondendo-Ihe uma idade média de 30.78 anos $(\mathrm{dp}=4,904)$. Todos os procedimentos éticos e legais foram assegurados. Os dados foram tratados com recurso ao SPSS versão 23.0 para Windows.

0 instrumento de recolha de dados foi o questionário que permitiu caracterizar a mostra a nível sociodemográfico (idade, estado civil, escolaridade, situação profissional, profissão e residência) e contexto de amamentação (primeira vez que amamenta, amamentação na 1a hora de vida, tipo de aleitamento após o parto e no internamento, experiência de amamentar, responsável pela decisão de amamentar, idade gestacional em que considerou a possibilidade de amamentar, fontes de informação sobre a amamentação, tipo de informação recebida relativa à amamentação, dificuldades rela- 


\section{DIFICULDADES NA AMAMENTAÇÃO NO PRIMEIRO MÊS DE VIDA: IMPACTO DOS CONTEXTOS DE VIDA}

cionadas com a amamentação, apoio dos enfermeiros nas dificuldades sentidas, dificuldades ultrapassadas, utilização de mamilo de silicone para amamentar, dúvidas sobre amamentação). 0 protocolo de colheita de dados inclui ainda a escala de satisfação com a vida de Diener et al. (1985) adaptada para a população portuguesa por Simões em 1992.

\section{RESULTADOS}

Relativamente à caracterização sociodemográfica, verificamos que a maioria das participantes $(88.6 \%)$ é casada ou vive em união de facto, tem como habilitações literárias 0 ensino superior $(42.0 \%)$. Mais de metade das participantes (61.2\%) encontra-se empregada em tempo completo, sendo relevante o percentual de mulheres desempregadas (29.8\%). A categoria profissional especialista de profissões intelectuais e científicas é verificada em $32.0 \%$ das participantes e $51.0 \%$ reside na cidade.

Quanto à caracterização do contexto de amamentação verificamos que $62.4 \%$ está a amamentar pela primeira vez, $84.5 \%$ refere ter amamentado na primeira hora de vida, $85.9 \%$ fez aleitamento materno exclusivo durante o internamento hospitalar, a quase totalidade da amostra $(96.8 \%)$ admite que esta está a ser uma experiência agradável, 86.3\% refere que a decisão de amamentar foi própria, $90.1 \%$ admite que a altura da gravidez em que considerou pela primeira vez a possibilidade de amamentar foi no primeiro trimestre, são os enfermeiros a principal fonte de informação relativamente ao aleitamento materno (89.8\%). Da informação recebida destacam-se as vantagens da amamentação (92.2\%), frequência das mamadas (84.3\%), posições para amamentar (83.1\%), prevenção das fissuras no mamilo (64.3\%), tratamento das fissuras (56.1\%). De entre as informações menos recebidas, destacam-se as que se referem à mastite.

Constata-se que $52.2 \%$ das participantes admitem não ter dificuldades na amamentação. As que referiram ter dificuldades com a amamentação $(n=132)$, a dificuldade mais frequente relacionava-se com as fissuras (41.7\%), seguindo-se as dificuldades na pega $(28.8 \%)$ e 0 ingurgitamento mamário $(16.7 \%)$. A quase totalidade da amostra $(91.1 \%)$ refere que as dificuldades associadas com a amamentação já foram ultrapassadas.

Verifica-se também que $21.6 \%$ das participantes admitem ter oferecido leite artificial ao bebé, das quais, $42.9 \%$ fizeram-no por considerarem que o bebé tinha fome, $26.8 \%$ justificam pela perda de peso. Os resultados revelam que a grande maioria das participantes (82.7\%) não 0 utiliza mamilo de silicone.

Os resultados apresentados indicam que a grande maioria (91.4\%) não possui dúvidas sobre a amamentação. Admitem que a experiência de amamentar ao $30^{\circ}$ dia de vida do bebé está a ser agradável. Quando questionadas acerca das dificuldades com a amamentação ao $30^{\circ}$ dia de vida do bebé, a maioria (53.4\%) admitiu que não as tem. Das participantes que afirmaram ter dificuldades, sobressaem $36.3 \%$ que apresentam dificuldades em relação às fissuras, seguindo-se aquelas com dificuldades na pega (31.9\%). Os enfermeiros, na grande maioria, apoiaram nas dificuldades $(97.0 \%)$. A quase totalidade da amostra (92.2\%) confirmou que ultrapassou as dificuldades iniciais associadas à amamentação.

Ao analisar a experiência de amamentar nos dois momentos de avaliação, verificamos que 97.5\% que avaliou esta experiência como agradável ao $7^{0}$ dia de vida do seu bebé continua a classificá-la como agradável ao $30^{\circ}$ dia, enquanto $60.0 \%$ das que consideraram esta uma experiência como agradável no primeiro momento de avaliação passaram a classificá-la de desagradável no segundo momento de avaliação. Por outro lado, $2.5 \%$ das participantes que admitem que esta foi uma experiência desagradável ao $7^{0}$ dia, já consideram agradável amamentar ao $30^{\circ}$ dia. Relativamente ao apoio prestado pelos enfermeiros, $97.3 \%$ admite ter tido apoio nas dificuldades 
sentidas ao $7^{\circ}$ e $30^{\circ}$ dia de vida do bebé. Constatamos também que $71.4 \%$ das participantes que tiveram apoio dos enfermeiros ao $7^{0}$ dia admitem não 0 ter ao $30^{\circ}$ dia.

Apuramos que $93.9 \%$ que admite ter ultrapassado as dificuldades com a amamentação ao $7^{0}$ dia de vida do bebé referem que as mesmas também foram ultrapassadas ao $30^{\circ}$ dia. Das mulheres que admitem não ter oferecido leite artificial ao seu bebé ao $7^{0}$ dia (94.8\%), também não o fizeram ao $30^{\circ}$ dia. Verificamos que $5.2 \%$ das participantes que ofereceram este tipo de leite ao bebé ao $7^{\circ}$ dia, deixaram de 0 oferecer ao $30^{\circ}$ dia.

Constatamos que $30.8 \%$ das participantes não possuíam dúvidas sobre a amamentação no primeiro momento, passando a tê-las aquando da segunda avaliação. Por fim, 69.2\% admitem ter dúvidas nos dois momentos de avaliação.

Na globalidade, as participantes ao $30^{\circ}$ dia de vida do seu bebé revelam-se mais satisfeitas com a vida $(M=20.22+/-3.862 d p)$, com significância estatística $(p=0.000)$. Pela matriz de correlação de Pearson estabelecida com o total da Escala da Satisfação com a Vida, nos dois momentos de avaliação verificamos que as correlações entre os dois momentos de avaliação são positivas e significativas, aferindo-se que a maiores índices da satisfação com a vida ao $7^{0}$ dia de vida do bebé correspondem maiores índices de satisfação com a vida ao $30^{\circ}$ dia de vida do bebé.

Quanto às dificuldades associadas à amamentação em função da escolaridade, verificamos foram encontradas diferenças estatísticas significativas $(p=0.000)$, onde o percentual mais elevado recaiu no grupo de puérperas com 0 ensino superior, as quais apresentam dificuldades na amamentação ao $7^{\circ}$ e $30^{\circ}$ dia de vida do bebé $(60.6 \%)$.

No que se refere às dificuldades na amamentação segundo o estado civil, $88.7 \%$ das casadas ou em união de facto não tinham dificuldades em nenhum dos dois momentos de avaliação.

Atendendo-se à variável situação profissional foram encontradas diferenças estatisticamente significativas $(p=0.034)$, referindo que no grupo de participantes sem dificuldades ao $7^{\circ}$ e $30^{\circ}$ dia de vida do bebé, há um predomínio das empregadas a tempo inteiro (55.7\%). Salientamos que $64.2 \%$ a trabalhar a tempo completo apresentam dificuldades nos dois momentos de avaliação.

Quanto à zona de residência, também foram encontradas diferenças estatisticamente significativas $(p=0.042)$, onde $51.5 \%$ das participantes residentes na cidade revelam não ter dificuldades relacionadas com a amamentação nos dois momentos de avaliação, seguindo-se os $39.2 \%$ das residentes na aldeia.

Sobressaem $62.4 \%$ das participantes que afirmam ser a primeira vez que amamentam, das quais $62.6 \%$ admitem não ter dificuldades associadas à amamentação, enquanto $62.1 \%$ apresentam-nas. Importa referir que 37.9\% que já têm experiência anterior de amamentação referem ter dificuldades, contrariamente a $37.4 \%$ das que admitem não ter dificuldades, todavia sem relevância estatística (X2=0.006; p. 0.937).

Há um claro predomínio de participantes que classificam a experiência de agradável (96.8\%), onde $100.0 \%$ não tem dificuldades associadas à amamentação e, apesar de estar a ser uma experiência agradável, para $93.8 \%$ há dificuldades na amamentação. As diferenças têm relevância estatística (X2=7.754; p. 0.005).

A quase totalidade das participantes $(90.1 \%)$ consideraram pela primeira vez a possibilidade de amamentar no primeiro trimestre de gravidez, observando-se que $85.4 \%$ não tem dificuldades associadas à amamentação e 94.6\% têm-nas, tendo-se registado diferenças estatisticamente significativas (X2=11.808; p. 0.008).

Quanto à superação das dificuldades associadas à amamentação, a maior representatividade é a das participantes que confirmam essa superação (91.1\%), sendo igualmente neste grupo onde registamos os percentuais mais expressivos em relação à inexistência de dificuldades na amamen- 
tação $(99.1 \%)$ e à presença das mesmas (83.8\%, com diferenças estatisticamente significativas $(X 2=17.765 ; p=0.000)$.

Na globalidade, as participantes ao $30^{\circ}$ dia de vida do seu bebé revelam-se mais satisfeitas com a vida. Fazendo-se uma análise tendo em conta os dois momentos de avaliação, constatamos que, no grupo de participantes sem dificuldades ao $7^{\circ}$ e $30^{\circ}$ dia de vida do bebé, prevalecem as que estão satisfeitas com a sua vida (40.2\%). Ressalvamos que são as participantes que referem estar satisfeitas com a vida que, em todos os momentos, se destacam.

\section{DISCUSSÃO DOS RESULTADOS}

Ainda que as evidências apontem para uma elevada incidência do aleitamento materno ao nascimento, continua a verificar-se que esta tendência diminui ao longo do primeiro mês de vida do bebé, indicando que um número significativo de mães não consegue cumprir o seu projeto de amamentar, resultando num abandono precoce da amamentação (Cunha, Santos \& Gonçalves, 2012). Importa referirmos que a decisão de amamentar é individual, todavia, interpolada com influências de socialização da mulher e com fatores motivacionais. A experiência da amamentação torna-se num momento delicado para as mulheres, cujas orientações adquiridas no processo gravídico nem sempre estão em consonância com a prática experienciada pelas mesmas, fazendo com que esta seja uma fase revestida de dúvidas e dificuldades, as quais poderão fazer com que a mulher se sinta mais vulnerável e insegura.

Os resultados obtidos comprovam que a prática de aleitamento materno, no período puerperal, acarreta aspetos críticos associados às dificuldades iniciais no estabelecimento da amamentação, designadamente problemas com a mama, ingurgitamento mamário, posicionamento e pega, ou problemas relacionados com a interação que podem exacerbar os primeiros (Pinto, 2008). Como tal, poderemos afirmar que a amamentação é determinada pela influência de vários fatores que abarcam os aspetos individuais, familiares, determinantes contextuais, sociodemográficos, bem como 0 papel que os profissionais de saúde têm no seu estabelecimento e manutenção (Venâncio \& Monteiro, 2006).

Verificámos que $62.4 \%$ das participantes está a amamentar pela primeira vez. A grande maioria $(84.5 \%)$ admitiu ter amamentado na primeira hora de vida, bem como observámos que $85.9 \%$ admite que o seu bebé foi exclusivamente amamentado com leite materno. Estes resultados corroboram os apurados por Gubert, J.K., Veieira, C.S., Oliveira, B.R.G., Delatore, S., \& Sanches, M.M. (2012), segundo os quais a grande maioria das mães estava a amamentar os seus filhos no final do primeiro mês, em aleitamento materno exclusivo. Constataram, de igual modo, que as puérperas mais novas são as que estão a vivenciar, pela primeira vez, a experiência de amamentação, classificando-a como agradável, o que foi verificado no nosso estudo, na medida em que quase a totalidade da amostra (96.8\%) admitiu que esta está a ser uma experiência agradável.

A decisão de amamentar foi por iniciativa própria (86.3\%). Para $90.1 \%$ das participantes a altura da gravidez em que considerou pela primeira vez a possibilidade de amamentar ocorreu no primeiro trimestre. Sandes, A., Nascimento, C., Figueira, J., Gouveia, R., Valente, S., Martins, S.,... Silva, L. J. (2007), no seu estudo verificou que $91 \%$ das mães amamentavam o seu filho, das quais $77,7 \%$ em exclusividade e por iniciativa própria.

Outros resultados relevantes referem-se às fontes de informação sobre amamentação, dos quais para a quase totalidade da amostra, sobressaíram os enfermeiros (89.8\%). Sousa e Bernardes (2010) também verificaram que o enfermeiro e o médico são as fontes de informação mais reiteradas na promoção do aleitamento materno exclusivo. As mães, cujas fontes de informação sobre a amamentação são, sobretudo, os profissionais de saúde e os familiares/amigos, amamentam duran- 
te mais tempo em relação às que têm unicamente os profissionais de saúde como fonte de informação.

Procurámos saber quais as dificuldades associadas à amamentação em função das variáveis sociodemográficas, tendo-se apurado que, no grupo de mães com idade igual ou inferior aos 30 anos, as evidências apontaram para a existência de mulheres com dificuldades nos dois momentos de avaliação, com predomínio das que tinham dificuldades ao $7^{0}$ dia após o nascimento (56.5\%), todavia sem dificuldades ao $30^{\circ}$ dia de vida do bebé, seguindo-se os $50.0 \%$ das participantes sem dificuldades ao $7^{\circ}$ dia, mas com dificuldades ao $30^{\circ}$ dia, sendo igualmente expressivo o percentual de $48.5 \%$ sem quaisquer dificuldades relacionadas com a amamentação nos dois momentos de avaliação. 0 tipo de dificuldades que se fizeram sentir mais nos dois momentos de avaliação foram 0 ingurgitamento mamário (66.7\%), mastite $(63.6 \%)$, dificuldades na pega $(82.4 \%)$ e dificuldades na posição e postura para amamentar (50.0\%). Estas dificuldades estão em consonância com a literatura, na medida em que muitas mães experimentam algumas destas dificuldades durante a amamentação, especialmente no início do seu processo (Walker, 2008). Essas dificuldades podem ser facilmente superadas com aconselhamento, assistência e suporte dos profissionais de saúde e da própria família (Walker, 2008). Outra conformidade encontrada refere-se aos resultados de Frota e Marcopito (2004) que também verificaram a existência de casos de puérperas com algum tipo de dificuldade durante a amamentação, desde o primeiro dia até ao $30^{\circ}$ dia de amamentação. No estudo de Frota e Marcopito (2004), a ocorrência mais elevada de dificuldades durante esse período de amamentação ocorreu em puérperas adolescentes, sobretudo ao $7^{0}$ dia de vida do seu bebé. Importa salientamos que Ferreira, Nelas e Duarte (2011) argumentam que as mães com idade materna igual ou superior a 35 anos constituem um fator de proteção para ao aleitamento materno até ao $12^{0}$ mês.

Quase a totalidade da amostra $(91.1 \%)$ referiu que as dificuldades sentidas em relação à amamentação foram ultrapassadas. Verificámos que $96.8 \%$ tiveram 0 apoio dos enfermeiros para ultrapassar as dificuldades. Assim, depreendemos que os profissionais de saúde, sobretudo os enfermeiros, envolvidos na assistência às puérperas devem estar atentos às necessidades e dificuldades apresentadas pelas mesmas, reforçando a importância da amamentação, ouvindo-as, oferecendo apoio e orientações pertinentes.

Constatámos que $54.4 \%$ das participantes que residem na cidade têm menos dificuldades na amamentação nos dois momentos de avaliação, enquanto $40.0 \%$ das puérperas residentes na vila/aldeia as apresentam.

Em conformidade com alguns autores, são vários os fatores sociodemográficos que parecem condicionar o sucesso da amamentação, dos quais se destacam a idade materna superior a 34 anos e um nível de escolaridade superior (Aguiar \& Silva, 2011). No nosso estudo, as mulheres com mais escolaridade e casadas ou em união de facto revelaram mais dificuldades nos dois momentos de avaliação, o que poderá sugerir que, independentemente das variáveis sociodemográficas, as mulheres no processo de amamentação poderão enfrentar dificuldades que, não sendo apoiadas por profissionais de saúde, podem resultar na descontinuidade do aleitamento materno. A educação para a gestão eficaz da amamentação, pelos profissionais de saúde nas primeiras semanas pósparto, é uma estratégia potenciadora da taxa de amamentação. É que a insegurança materna é uma das maiores dificuldades para se iniciar com êxito a amamentação, fundada muitas vezes no desconhecimento das suas possibilidades de amamentar e em que a tendência natural é desistirem à menor dificuldade.

Os resultados demonstraram que $80.8 \%$ das participantes não possuíam dificuldades na amamentação ao $7^{0}$ dia de vida do bebé, continuando a não revelá-las no $30^{\circ}$ dia, enquanto $19.3 \%$ não as tinham no $7^{0}$ dia de vida do seu bebé, mas passaram a tê-las no $30^{\circ}$ dia. Estas evidências 
demonstram a necessidade de os enfermeiros fazerem uma reflexão sobre as suas práticas e formação sobre aleitamento materno, pois o papel do profissional de saúde é de educação, encorajamento e apoio, sempre tendo em conta as sugestões da mãe, preocupações e fundo cultural (Gatti, 2008).

A amamentação poderá parecer natural, todavia é dificultada pela realidade sociocultural em que a mulher está inserida e, por vezes, não é fácil para as mesmas exporem os seus sentimentos e as suas dificuldades face à amamentação. Todavia, há soluções para minimizar as dificuldades sentidas pelas mulheres durante a amamentação. Estas devem saber identificar o problema que está a ocorrer, seja ao $7^{0}$ dia, seja ao $30^{\circ}$ dia, e agir com rapidez nos primeiros sinais, porque se não ocorrer a reversão da situação, as dificuldades poderão agravar-se e a amamentação será prejudicada, colocando em risco a continuidade do aleitamento materno (Levy \& Bértolo, 2012).

Predominam as participantes satisfeitas com a vida. Estes resultados podem ser justificados com 0 facto de as mulheres, ao serem mães, estarem a realizar um objetivo de vida, traduzindo-se em melhor satisfação com a vida. Tendo-se como ponto de referência que a satisfação com a vida reflete o bem-estar individual, isto é, o modo e os motivos que levam as pessoas a viverem as suas experiências de vida de uma forma positiva, as participantes poderão ter uma perceção satisfatória da sua vida, sobretudo quando possuem uma rede de suporte social. Deste modo, podemos inferir que as participantes poderão demonstrar estar a experienciar um bem-estar subjetivo, decorrente de estados afetivos positivos. Neste sentido, reforçamos a ideia de que é importante ter 0 apoio dos profissionais de saúde disponíveis para escutar e ajudar a solucionar as dúvidas e os problemas que possam surgir, resultando, por inerência, em mais satisfação com a vida.

\section{CONCLUSÃO}

Sugerimos um maior apoio por parte dos profissionais de saúde, não só desde o início da gravidez onde a decisão de amamentar se manifesta, como também durante o primeiro mês de vida do bebé, onde as maiores dificuldades aparecem. A continuidade do acompanhamento assume-se como indispensável para se diminuir o abandono da amamentação precocemente. 0 apoio dos profissionais de saúde às mulheres que amamentam tem demonstrado uma concordância sobre os benefícios do aleitamento materno e atitudes muito positivas por parte das mulheres. Estes profissionais desempenham um papel importante na promoção e apoio ao aleitamento materno e promovem não só o conhecimento relativo à amamentação e as habilidades, como também, influenciam atitudes positivas proporcionando e incentivando 0 uso das estruturas de apoio à amamentação durante a gravidez e pós-parto.

\section{BIBLIOGRAFIA}

Aguiar, H., \& Silva, A. I. (2011). Aleitamento materno: A importância de intervir. Acta Médica Portuguesa, 24, 889-896. Acedido em http://www.actamedicaportuguesa.com/ revista/index.php/amp/article/.../1164

Cremonese, L., Wilhelm, L. A., Santos, C., Alves, C. N., Martello, N. V., Silva, S. C.,... Ressel, L. B. (2011). Dificuldades vividas no processo do aleitamento materno. Acedido em http://www.unifra.br/eventos/sepe2012/Trabalhos/5753.pdf

Cunha, A. C., Santos, C., \& Gonçalves, R. M. (2012). Concepções sobre maternidade, parto e amamentação em grupo de gestantes. Arquivos Brasileiros de Psicologia, 64(1), 139-155.

Ferreira, M., Nelas, P., \& Duarte, J. (2011). Motivação para 0 aleitamento materno: Variáveis intervenientes. Millenium, 40, 23-38.

Frota, D. A. L., \& Marcopito, L. F. (2004). Breastfeeding among teenage and adult mothers in Brazil. Revista Saúde Pública, 38(1), 85-92. 
Gatti, L. (2008). Maternal perceptions of insufficient milk supply in breastfeeding. Journal of Nursing Scholarship, 40(4), 355-363.

Greiner, T. (2014). Possibilidades e limitações da amamentação entre mulheres trabalhadoras formais. Revista Saúde Pública, 31(2), 149-156.

Gubert, J. K., Veieira, C. S., Oliveira, B. R. G., Delatore, S., \& Sanches, M. M. (2012). Avaliação do aleitamento materno de recém-nascidos prematuros no primeiro mês após a alta. Ciencia, Cuidado e Saúde, 11(1), 146-155.

Levy, L., \& Bértolo, H. (2012). Manual de aleitamento materno. Comité Português para a UNICEF/Comissão Nacional Iniciativa Hospitais Amigos dos Bebés.

Pinto, T. (2008). Promoção, protecção e apoio ao aleitamento materno na comunidade Revisão das estratégias no período pré-natal e após a alta. Arquivos de Medicina, 22, 2-3.

Sandes, A., Nascimento, C., Figueira, J., Gouveia, R., Valente, S., Martins, S.,... Silva, L. J. (2007). Aleitamento materno: Prevalência e factores condicionantes. Acta Médica Portuguesa, 20, 193200. Acedido em http://repositorio.ul.pt/bitstream/10451/17639/1/aleitamento_materno.pdf

Simões, A. (1992). Ulterior validação de uma escala de satisfação com a vida (SWLS). Revista Portuguesa de Pedagogia, 26, 503-515.

Sousa, N., \& Bernardes, A. (2010). Aleitamento materno: Prevalência e caracterização da informação prestada. Revista Portuguesa de Clínica Geral, 26(5), 440-448. Acedido em http://www.rpmgf.pt/ojs/index.php/rpmgf/article/view/10778

Venâncio, S. I., \& Monteiro, C. A. (2006). Individual and contextual determinants of exclusive breastfeeding in São Paulo, Brazil: A multilevel analysis. Public Health Nutrition, 9, 40-46.

Walker, M. (2008). Conquering common breast-feeding problems. Journal of Perinatal \& Neonatal Nursing, 22(4), 267-274. 
